مجلة جامعة سبها للعلوم البحتة والتطبيقية

Sebha University Journal of Pure \& Applied Sciences

Journal homepage: www.sebhau.edu.ly/journal/index.php/jopas

\title{
Study on loss processes in solar cells
}

Ahmed B Abdurrhman, Fatima Zakria, Hamed A. Said

Department of Electrical and Electronic Engineering, Faculty of Engineering Sciences and Technology, Sebha University, Libya

\section{Keywords:}

Photovoltaic (PV)

Intrinsic Losses

Extrinsic Losses

Full Thermal Model FTM

\begin{abstract}
A B S T R A C T
Determining heat sources for solar cells is essential to avoid energy loss, which in turn causes the efficiency of solar cells to decrease and therefore, the loss processes have a significant impact on solar conversion. This paper presents a study of intrinsic and exogenous losses in solar cells, identification of the resulting energy loss at different temperatures, and discusses the impact of exogenous and spectral reflectivity on solar cell performance. The results show an increase in thermal loss with an increase in temperature, which in turn leads to a decrease in the efficiency of solar cells. Also explained that the external radiate efficiency, spectral reflectance and operating temperature significantly affect the loss processes. The efficiency of the cell begins to decrease with the decrease of its external radiate efficiency.
\end{abstract}

$$
\text { دراسـة الفقد في الخلايا الشمسية بوسية عبد الرحمن، فاطمة زكريا، حامد عبد الحق سعيد }
$$$$
\text { قسم الهندسة الكهربائية والالكترونية ، كلية العلوم الهندسية والتقنية، جامعة سبها، ليبيا }
$$

الكلمات المفتاحية:

Photovoltaic (PV)

Intrinsic Losses

Extrinsic Losses

Full Thermal Model FTM

\section{الملخصص}

$$
\begin{aligned}
& \text { تحديد مصادر الحرارة للخلايا الشمسية يعد أمرا ضروريا لتجنب فقدان الطاقة الذي بدوره يسبب في خفض }
\end{aligned}
$$

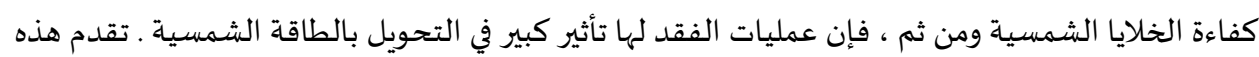

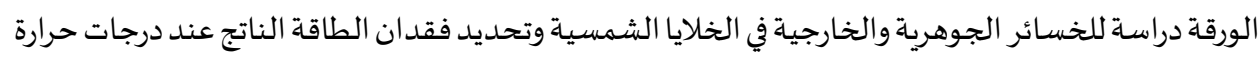

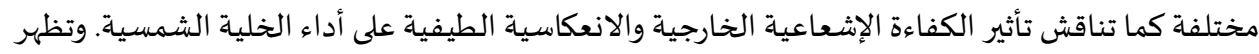

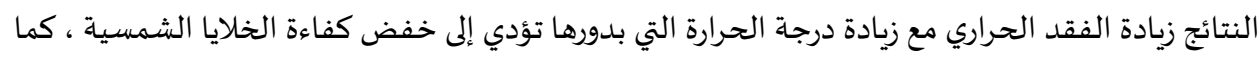

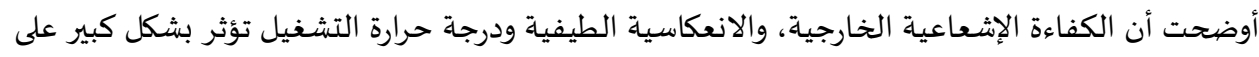

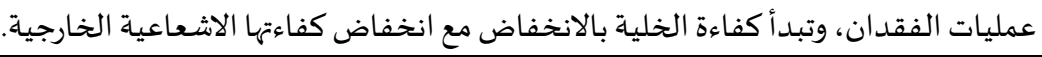

\section{Introduction}

Solar energy photovoltaic technology has developed rapidly for the past years and researchers over the world have been working hard on improving the efficiency and reducing the cost of photovoltaic devices. We constantly see new records of solar photovoltaic (PV) cell efficiencies, which are approaching the theoretical limits. However, the efficiency of photovoltaic devices grows slowly in recent years [1], [2].

Many studies hase been conducted on the factors that limit the efficiency of photovoltaic devices. In 1996, Shockley and Queasier developed the first detailed model for ideal solar cells that illustrated the mechanisms of intrinsic (internal) loss called the detailed equilibrium model [3]. Moreover, in 1980, proposed Henry a graphical method for understanding intrinsic losses [4], and in the same year, Wurfel published thermodynamic limitations of solar energy conversion based on the second law of thermodynamics, which is theoretically the highest efficiency of photovoltaic devices
[5]. Later on, studies wer carried out to investigate the coefficients of crystalline silicon radiate recombination [6] and Auger recombination [7]. Hirst studied five mechanisms of quantum intrinsic losses and gave a mathematical and graphical demonstration [8]. one year later, Auger recombination was quantified with an improved Model [9]. In addition, the intrinsic losses model for singlejunction solar cells was applied but this time considering the AM1.5g standard solar spectrum as the incident power [10]. As in 2015, the Non-Radiate-Recombination processes are considered in the analysis [11]. A year later, was developed full thermal model based on explicit formulas for calculating heat sources, and optical losses and internal processes of solar cells under any incident spectral irradiation [12]. The SQ limit is studied for ideal solar cells but this time using monochromatic light [13]. and has recently introduced an extension of the Full thermal model to evaluate the PV devices [1], As full thermal model was applied to a bifacial hetero junction solar cell in

*Corresponding author:

E-mail addresses: Ahm.ishteewi@ @ebhau.edu.ly, (F. Zakria)Fa.zakrya@ sebhau.edu.ly, (H. A. Said) Hmdhsd54@gmail.com Article History : Received 24 December 2020 - Received in revised form 13 February 2021 - Accepted 24 February 2021 
2019 [14] .

This paper studies the loss processes in photovoltaic devices depending on different kinds of parameters, such as external radiate efficiency(ERE), spectral reflectance, and operating temperature. Energy distributions of a crystalline silicon(c-Si) solar cell are presented to characterize the intrinsic and extrinsic losses in detail, calculated by a thermal model based on the model proposed by Dupré et al [12].

\section{METHODOLOGY}

Loss processes in solar cells consist of two parts: intrinsic losses (fundamental losses) and extrinsic losses. Intrinsic losses are unavoidable in single bandgap solar cells, even if in the idealized solar cells [8]. In this paper, this loss was determined based on the full thermal model (FTM) which includes the main loss mechanisms inside the cell and they are in detail explained in [1], [12]. In Table 1, briefly the explicit formulas to calculate different mechanisms and losses at Maximum Power Point (MPP), where the heat sources are represented as "Q" and non-heat sources as "P" ..

Table 1: Explicit formulas of FTM.

\begin{tabular}{|c|c|}
\hline Mechanism & Explicit Formula \\
\hline Reflection & $P_{\text {reflection }}=\int_{0}^{\infty} R_{c} P F D(E) E d E$ \\
\hline Below $\mathrm{E}_{\mathrm{s}}$ & $\begin{array}{l}Q_{\text {Heeliaw: }}= \\
\qquad \int_{0}^{E_{\theta}}\left(1-R_{c}-T_{c}\right) P F D(E) E d E\end{array}$ \\
\hline Thermalization & $\begin{array}{l}Q_{\text {ihermalination - }}^{\infty} \\
\int_{E_{0}}^{\infty}\left(1-R_{c}-T_{c}\right) P F D(E)\left(E-E_{g}\right) d E\end{array}$ \\
\hline Camot & $\mathrm{Q}_{\text {arrock }}=\mathrm{E}_{B}\left(1-\frac{\mathrm{T}_{a}}{T_{s}}\right)$ \\
\hline Angle Mismatch & $Q_{\text {angle mismatch }}=J$ MPP $\left(\frac{k T_{a}}{q}\right) \ln \left(\frac{\Omega_{\text {emit }}}{\Omega_{a b s}}\right)$ \\
\hline$R R$ - Emission & $P_{\text {smissiononv }}=E_{g} J_{\text {emissionsop }}$ \\
\hline $\begin{array}{l}\text { NRR Voltage } \\
\text { Drop }\end{array}$ & $Q_{\text {NRRAV }}=J$ MPP $\left(\frac{k T_{a}}{q}\right) \ln \left(\frac{1}{E R E M P P}\right)$ \\
\hline $\begin{array}{l}\text { NRR Current } \\
\text { Drop }\end{array}$ & $Q_{\text {NRRAl }}=E_{g}\left(\frac{1}{E R E \text { MPP }}-1\right)$ Jemissionhep \\
\hline Transport & $Q_{\text {trunsport }}=R_{s} J_{M P P}^{2}$ \\
\hline Shunt & $Q_{\text {shuxt }}=\frac{E_{g}}{q}\left(\frac{V_{M P P}+J M P P}{R_{S}}\right)$ \\
\hline Electrical Power & $P_{M P P}=V_{M P P} J_{M P P}$ \\
\hline The total heat & 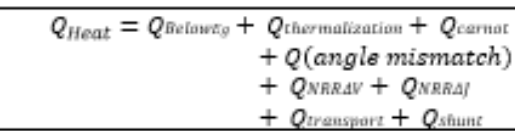 \\
\hline
\end{tabular}

The model was applied of crystalline silicon(c-Si) solar cell with the bandgap of $1.1246 \mathrm{eV}$, under an AM1.5 solar illumination (photon flux density $=1000.25 \mathrm{~W} / \mathrm{m} 2$ ) The maximum output current and voltage density was determined numerically using:

$$
J(V)=J_{s c, \text { Max }}-\frac{1}{E R E} J_{\text {emission }} \exp \left(\frac{q\left(V+J R_{s}\right)}{k T_{a}}\right)-\frac{V+J R_{s}}{R_{s h}}
$$

Where Jemit refers to the photocurrent loss caused by radiative recombination, defined as:

$$
J_{\text {emission }}=\frac{2 \mathrm{q} \Omega_{\text {emit }}}{c^{2} h^{3}}\left(E^{2} k T_{a}+2 E k^{2} T_{a}^{2}+2 k^{3} T_{a}^{3}\right) \exp \left(\frac{q V-E}{k T_{a}}\right)
$$

To discuss the intrinsic losses of solar cells quantitatively, the reflectance $R_{-} c$ and the transmittance $T \_c$ of the cell are set to zero, ERE is set to 1, and resistances of the cell (R_s and R.sh) are ignored. Then eq. (1) is written as:

$$
J(V)=J_{s c, \text { Max }}-\frac{1}{E R E} J_{\text {emission }} \exp \left(\frac{q(V)}{k T_{a}}\right)
$$

Fig. 1 shows the equivalent circuit diagram of a solar cell. By taking all loss processes into consideration, eq. (1) is used to derive the necessary parameters. Firstly,V_MPPand J_MPPare calculated by finding the maximum power point $\left(\mathrm{P}_{-}\right.$MPP $\llbracket=\mathrm{J}_{-}$MPP $\left.\mathrm{V} \rrbracket \_\mathrm{MPP}\right)$ with the variables imposed $\mathrm{R} \_\mathrm{s}=1.1 \Omega \llbracket \mathrm{cm} \rrbracket \wedge 2, \mathrm{R} \_\mathrm{sh}=240 \Omega \llbracket \mathrm{cm} \rrbracket$ 22, T_c $=0, T_{-} \mathrm{s}=5800 \mathrm{~K}, \bar{\Omega} \Omega_{-} \mathrm{emit}=\pi, \Omega$ abs $=\Omega_{-}$emit 46200 , and

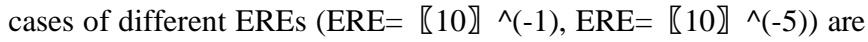
calculated to illustrate the impact of external radiate efficiency on loss processes at different temperatures, as well $\mathrm{R} \_c=0.1, \mathrm{R} \_\mathrm{c}=0.05$ to illustrate the effect of spectral reflectance on cell performance.

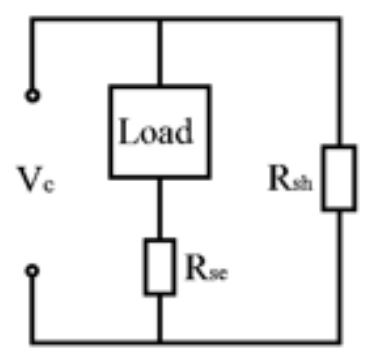

Fig. 1. Equivalent circuit diagram of a solar cell showing the load, series resistance (R_s), shunt resistance (Rsh) and the voltage across the cell (V_C).

\section{Results and discussion}

The results in Table 2 and Fig. 2 indicate the fundamental (basic) losses that they take up the majority of the incident energy, and that a large amount of solar energy is converted into heat. The efficiency of the ideal crystalline silicon cell $(E R E=1)$ is achieved $33.52 \%$ at a temperature $298.15 \mathrm{~K}$. The relative error is

$$
\delta=(\mid \text { POutput }+ \text { PHeat }+ \text { PEmission }- \text { PIncident } \mid) / \text { PIncident. (4) }
$$

The total of energy distributions expressed in this equation agree well with the incident solar energy, which prove the model of high accuracy.

Table 2 Energy distribution and corresponding parameters of a c-Si solar cell

\begin{tabular}{|c|c|c|c|c|}
\hline \multicolumn{5}{|c|}{ crystalline silicon solar cell, $\mathrm{E}_{\mathrm{a}}=1.1246 \mathrm{eV}, \mathrm{Ta}=298.15 \mathrm{k}$} \\
\hline & Current & Voltage & Power & $/ \mathrm{P}_{\text {Incident }}$ \\
\hline & $\mathrm{A} / \mathrm{m}^{2}$ & $\mathrm{~V}$ & $\mathrm{~W} / \mathrm{m}^{2}$ & $\%$ \\
\hline $\mathrm{J}_{\mathrm{ph}} / \mathrm{V}_{\text {mar }} \mathrm{P}_{\text {Inodant }}$ & 436.40 & 1.1246 & 1000.25 & 100 \\
\hline $\mathrm{Q}_{\text {Below }}$ & & & 194.34 & 19.45 \\
\hline $\mathrm{Q}_{\text {Thermalization }}$ & & & 315.35 & 31.51 \\
\hline $\mathrm{P}_{\text {Emission }}$ & 13.13 & & 14.76 & 1.47 \\
\hline $\mathrm{Q}_{\text {Camot }}$ & & 0.0578 & 24.45 & 2.44 \\
\hline $\mathrm{Q}_{\text {Angle }}$ & & 0.276 & 116.80 & 11.68 \\
\hline $\mathrm{P}_{\text {Output }}$ & $\mathrm{J}_{\mathrm{MPp}}=$ & $\mathrm{V}_{\mathrm{MPp}}=$ & 335.24 & $33.52(\eta)$ \\
\hline$\Delta$ & 423.27 & 0.792 & & 0 \\
\hline$Q_{\text {Heat }}$ & 0 & 0 & -0.6 & 0 \\
\hline
\end{tabular}

At room temperature.

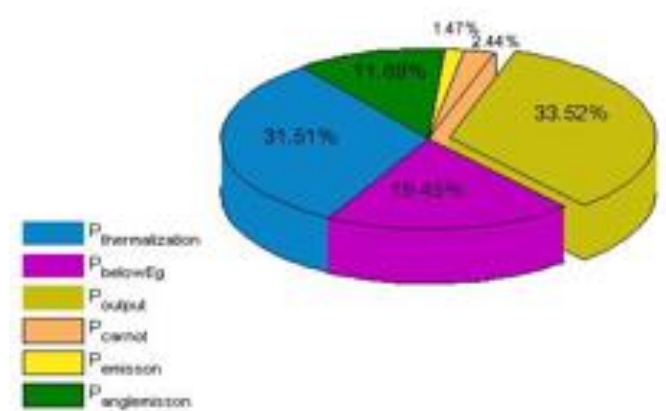

Fig. 2. Energy distributions of a c-Si solar cell at room temperature.

Fig. 3 shows the effect of external radiation efficiency on the efficiency of solar cells below the solar spectrum (AM1.5) at a 
temperature (298.15) as the highest efficiency obtained at ERE $=1$ and cell efficiency begins to decrease with lower external radiation efficiency. Fig. 4 shows the distribution of fallen solar energy (imposing black body radiation at $5800 \mathrm{k}$ ) at different external radiate efficiency with the bandgap, the larger the bandgap, the smaller the heat source and the fewer loss processes associated with radiation reunion with increased band gap and increased external radiate efficiency. From Table 3 for external radiate efficiency it does not affect loss processes except for NRR loss where NRR_V $=2.07 \%$ at $\mathrm{ERE}=\llbracket 10 \rrbracket \wedge(-1)$ and $10.44 \%$ at $\mathrm{ERE}=\llbracket 10 \rrbracket \wedge(-5)$ and At $\mathrm{ERE}=2.22 * \llbracket 10 \rrbracket \wedge(-7)$ is $13.75 \%$ and this excess energy is converted to heat, which reduces cell efficiency as a result.

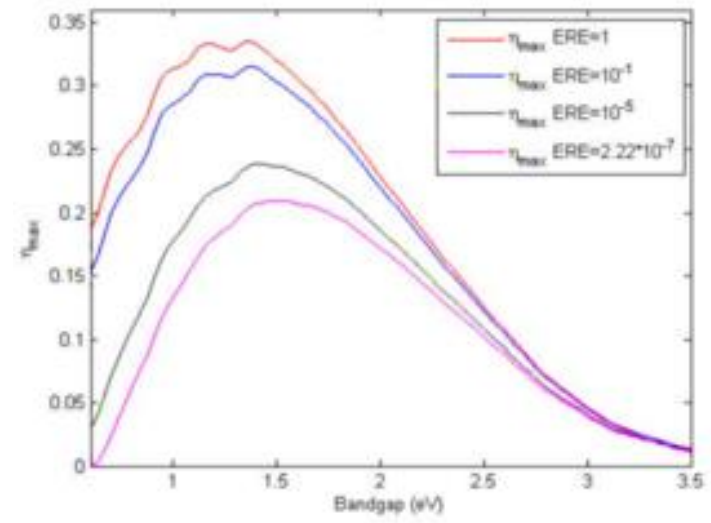

Fig. 3. Efficiency of ideal solar cells only considering the intrinsic losses at different external radiate efficiency at room temperature.
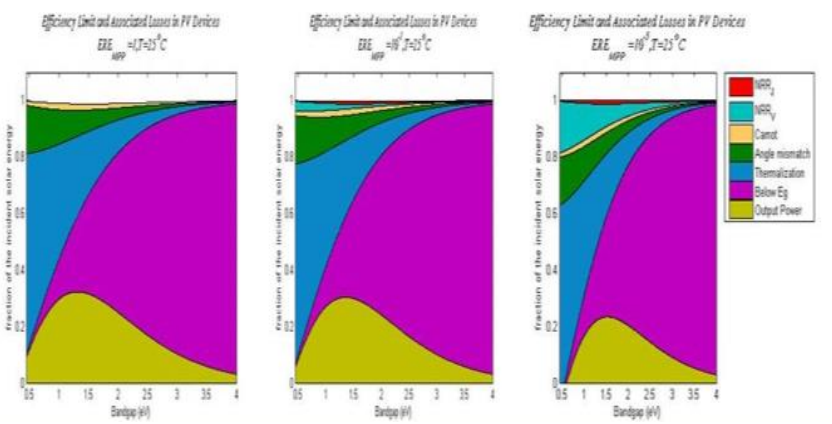

Fig. 4. Energy distribution of solar cells in radiate limit at room temperature. The external radiate efficiency are $\mathrm{ERE}=1$ in (a), $\mathrm{ERE}=10-1$ in (b), $\mathrm{ERE}=10-5$ in (c), respectively.

The results in the Table 4, Table 5 and the fig. 5 ,fig. 6 showed that an increase in the temperature of the cell leads to a decrease in its efficiency from $16.68 \%$ at room temperature to $14.58 \%$ at $55^{\circ}$ at the radiative limit $\mathrm{ERE}=\llbracket 10 \rrbracket \wedge(-5)$, and from $24.4 \%$ at room temperature to $23.1 \%$ at $55^{\circ}$ at the radiative limit ERE $=\llbracket 10 \rrbracket \wedge(-1)$. Also showed the increase in the amount of heat generated from $705.12 \mathrm{~W} \mathrm{~m}^{\wedge}(-2)$ at room temperature to $760.81 \mathrm{~W} \mathrm{~m}^{\wedge}(-2)$ at $55^{\circ}$. This is humiliating that the amount of heat is directly proportional to the high temperature and inversely to external radiate efficiency (ERE) . To study the effect of spectral reflectance on the performance of the solar cell, the results shown in Table 6 and fig. 7 show an increase in efficiency from $24.4 \%$ at $\mathrm{R} \_\mathrm{c}=0.1$ to $25.85 \%$ at $\mathrm{R} \_\mathrm{c}=$ 0.05 in room temperature, this increase is a decrease in the process of loss of the current by reflection from $43.64\left(\mathrm{~A} \square \mathrm{m}^{\wedge} 2\right)$ at $\mathrm{R} \_\mathrm{c}=0.1$ to $21.82 \mathrm{~A} / \mathrm{m}^{\wedge} 2$ at $\mathrm{R} \_\mathrm{c}=0.05$.

Table 3 Energy distribution and corresponding parameters of a c-Si solar cell with different external radiate efficiency at room temperature.

\begin{tabular}{|c|c|c|c|c|c|c|c|c|c|c|c|c|}
\hline \multicolumn{13}{|c|}{ crystalline silicon solar cell, $\mathrm{E}_{\mathrm{g}}=1.1246 \mathrm{eV} . \mathrm{Ta}=298.15 \mathrm{~K}$} \\
\hline & \multicolumn{4}{|c|}{ ERE $=10^{-1}$} & \multicolumn{4}{|c|}{ ERE $=10^{5}$} & \multicolumn{4}{|c|}{ ERE $=2.22 \div 10^{-7}$} \\
\hline & Current & Voltage & Power & $\mid P_{\text {trindatat }}$ & \begin{tabular}{|l|l|} 
Current \\
\end{tabular} & Voltage & Power & $\mid \mathbb{P}_{\text {thidetat }}$ & \begin{tabular}{|l|} 
Current \\
\end{tabular} & \begin{tabular}{|l|l} 
Voltage \\
\end{tabular} & \begin{tabular}{|l|} 
Powerer \\
\end{tabular} & $\mathbb{P}_{\text {thideter }}$ \\
\hline Units & $\mathrm{A} / \mathrm{m}^{2}$ & $\mathrm{v}$ & $\mathrm{W} / \mathrm{m}^{2}$ & $\%$ & $A / \mathrm{m}^{2}$ & $\mathrm{v}$ & $\mathrm{W} / \mathrm{m}^{2}$ & $\%$ & $\mathrm{~A} \mathrm{~m}^{2}$ & $\mathrm{v}$ & $\mathrm{W} / \mathrm{m}^{2}$ & $\%$ \\
\hline 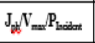 & 436.4 & 1.124 & 1000.25 & 100 & 436.4 & 1.124 & 1000.25 & 100 & 436.4 & 1.124 & 1000.25 & 100 \\
\hline Preselosen & 43.64 & & 100.0 & 10.00 & 43.64 & & 100.0 & 10.00 & 43.64 & & 100.0 & 10.00 \\
\hline $\mathrm{P}_{\text {Bebrr }}$ & & & 174.9 & 17.49 & & & 174.9 & 17.49 & & & 174.9 & 17.49 \\
\hline $\mathrm{P}_{\text {Thenewivision }}$ & & & 283.81 & 28.38 & & & 283.81 & 28.38 & & & 283.8 & 28.38 \\
\hline $\mathrm{P}_{\text {Eminisina }}$ & 1.11 & & 1.24 & 0.12 & 0 & & 0 & 0 & 0 & & 0 & 0 \\
\hline$P_{\text {cranot }}$ & & 0.057 & 20.29 & 2.02 & & \begin{tabular}{|l|}
0.051 \\
\end{tabular} & 20.40 & 2.04 & & \begin{tabular}{|l}
0.057 \\
\end{tabular} & 20.18 & 2.01 \\
\hline$P_{\text {Alplo }}$ & & \begin{tabular}{|l|l|}
0.276 \\
\end{tabular} & \begin{tabular}{|l|}
96.91 \\
\end{tabular} & 9.69 & & 0.276 & $\begin{array}{l}97.46 \\
\end{array}$ & 9.74 & & 0.276 & 96.40 & 9.64 \\
\hline $\mathrm{P}_{\text {NRR }}$ & 10.01 & & $\begin{array}{ll}11.24 \\
\end{array}$ & 1.12 & 18.42 & & \begin{tabular}{|l|l|}
20.71 \\
\end{tabular} & 2.07 & \begin{tabular}{|l|}
25.79 \\
\end{tabular} & & \begin{tabular}{|l|}
29.20 \\
\end{tabular} & 2.92 \\
\hline $\mathrm{P}_{\mathrm{NRR}, \mathrm{Y}}$ & & \begin{tabular}{|l|}
0.059 \\
\end{tabular} & 20.77 & 2.07 & & 0.295 & 104,47 & 10.44 & & 0.393 & \begin{tabular}{|l|}
137.51 \\
\end{tabular} & 13.75 \\
\hline $\mathrm{P}_{\text {stip }}$ & & 0.035 & 12.33 & 1.23 & & 0.0353 & 12.47 & 1.24 & & \begin{tabular}{|l|l|}
0.034 \\
\end{tabular} & 12.20 & 1.22 \\
\hline$P_{\text {shat }}$ & 30.47 & & 34.25 & 3.43 & 21.15 & & 23.77 & 3.37 & \begin{tabular}{|l|}
17.44 \\
\end{tabular} & & \begin{tabular}{|l|}
19.60 \\
\end{tabular} & 1.96 \\
\hline $\mathrm{P}_{\text {Oaput }}$ & $\begin{array}{c}\text { JMPP } \\
=351.17\end{array}$ & $\begin{array}{l}\text { TIPPP } \\
=0.696\end{array}$ & 244.48 & $\begin{array}{c}24.44 \\
(\eta)\end{array}$ & $\begin{array}{c}\text { JMPP } \\
=353.18 \\
\end{array}$ & $\begin{array}{l}\text { TIPPP } \\
=0.472\end{array}$ & 166.81 & $16.68(\eta)$ & \begin{tabular}{|c}
$\mathrm{JMPP}$ \\
$=349.34$
\end{tabular} & $\begin{array}{l}\text { WMPP } \\
=0.383\end{array}$ & 134.04 & 13.40(n) \\
\hline$\Delta$ & 0 & 0 & 0.03 & 0 & 0.01 & 0 & 4.5 & -0.45 & 0.19 & \begin{tabular}{|l|} 
\\
\end{tabular} & -7.6 & 0.76 \\
\hline$P_{\text {Heat }}$ & & 654.5 & & 65.4 & & 705.12 & & 70.51 & & 773.79 & & 77.37 \\
\hline
\end{tabular}

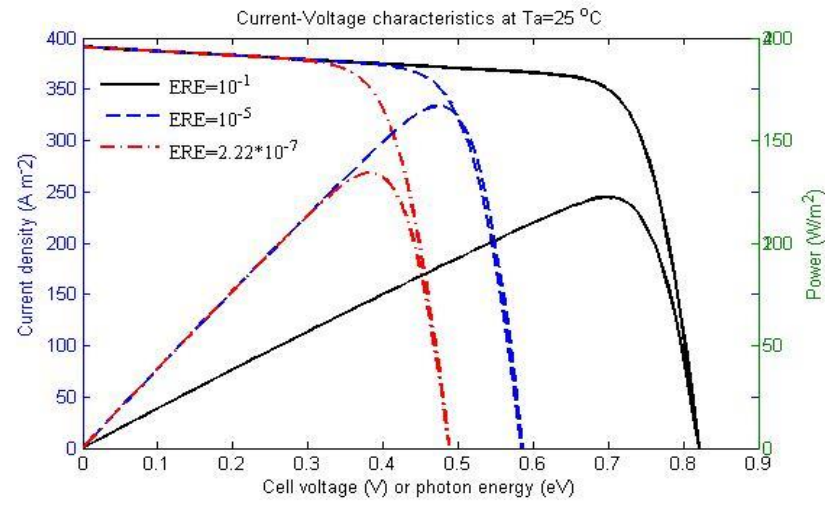

Fig. 5. Voltage-current curve of the solar cell at different external radiative efficiencies.

Table 4 Energy distribution and corresponding parameters of a c-Si solar cell with external radiate efficiency (ERE) $=10-1$ at different temperatures.

\begin{tabular}{|c|c|c|c|c|c|c|c|c|c|c|c|c|}
\hline \multicolumn{13}{|c|}{ crystalline silicon solar cell, $\mathrm{E}_{\mathrm{g}}=1.1246 \mathrm{EV}$} \\
\hline \multicolumn{13}{|c|}{$\mathrm{ERE}=10^{-1}$} \\
\hline & \multicolumn{4}{|c|}{$\mathrm{T}_{\mathrm{a}}=308.15 \mathrm{~K}$} & \multicolumn{4}{|c|}{$\mathrm{T}_{\mathrm{a}}=318.15 \mathrm{~K}$} & \multicolumn{4}{|c|}{$\mathrm{Ta}=328.15 \mathrm{~K}$} \\
\hline & Current & Voltage & Power & Prindent & Current & Voltage & Power & P Precident & Current & Voltage & Power & P Placidat \\
\hline Units & A'm 2 & $\mathrm{v}$ & $\mathrm{W} / \mathrm{m} 2$ & 96 & $A^{A} \mathrm{~m} 2$ & $\mathrm{v}$ & $w / m 2$ & 96 & $\mathrm{~A} / \mathrm{m} 2$ & $\mathrm{v}$ & $\mathrm{W} / \mathrm{m} 2$ & 96 \\
\hline 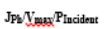 & \begin{tabular}{|l|l|}
436.4 \\
\end{tabular} & 1.124 & 1000.25 & 100 & \begin{tabular}{|l|l|}
436.4 \\
\end{tabular} & 1.124 & 1000.25 & 100 & 436.4 & 1.124 & 1000.25 & 100 \\
\hline$P_{\text {Reflection }}$ & $\mid 43.64$ & & 100.0 & 10.00 & 43.64 & & 100.0 & 10.00 & 43.64 & & 100.0 & 10.00 \\
\hline $\mathbf{P}_{\text {Below }}$ & & & 174.9 & 17.49 & & & 174.9 & 17.49 & & & 174.9 & 17.49 \\
\hline PThermalization & & & 283.1 & 28.31 & & & 283.1 & 28.31 & & & |283.1 & 28.31 \\
\hline$P_{\text {Emission }}$ & 1.31 & & 1.4 & 0.14 & 0.43 & & 0.48 & 0.04 & 0.40 & & 0.44 & 0.04 \\
\hline$P_{\text {Carnot }}$ & & 0.059 & 20.8 & 2.08 & & 0.061 & 21.4 & 2.14 & & 0.063 & 22.2 & 2.22 \\
\hline$P_{\text {Angle }}$ & & 0.285 & 99.6 & 9.96 & & 0.294 & 102.3 & 10.23 & & 0.303 & 106.3 & 10.63 \\
\hline PNRR J $_{\text {N }}$ & 11.87 & & 13.3 & 1.33 & $\mid$\begin{tabular}{|l}
$\mid$ \\
\end{tabular} & & 15.6 & 1.56 & 12.41 & & 13.9 & 1.39 \\
\hline PNRR-V & & 0.061 & 21.3 & 2.13 & & 0.063 & 21.9 & 2.19 & & 0.059 & 22.7 & 2.27 \\
\hline$P_{\text {Series }}$ & & 0.035 & 12.2 & 1.22 & & 0.0348 & 12.08 & 1.20 & & 0.035 & 12.25 & 1.22 \\
\hline$P_{\text {Shunt }}$ & 30.08 & & 33.8 & 3.38 & 29.67 & & 33.35 & 3.33 & 28.95 & & 32.55 & 3.25 \\
\hline Poutput & $\begin{array}{l}\text { JMPP } \\
=349.5\end{array}$ & $\begin{array}{l}\text { VMPP } \\
=0.686\end{array}$ & 240.03 & $\begin{array}{c}24.0 \\
(\eta)\end{array}$ & $\begin{array}{l}\text { JMPP } \\
=347.6\end{array}$ & $\begin{array}{l}\text { VMPP } \\
=0.677\end{array}$ & 235.47 & $23.54(\eta)$ & \begin{tabular}{|c|c|} 
JMPP \\
$=350.01$
\end{tabular} & $\begin{array}{l}\text { VMPP } \\
=0.66\end{array}$ & 231.02 & $23.10(1)$ \\
\hline
\end{tabular}




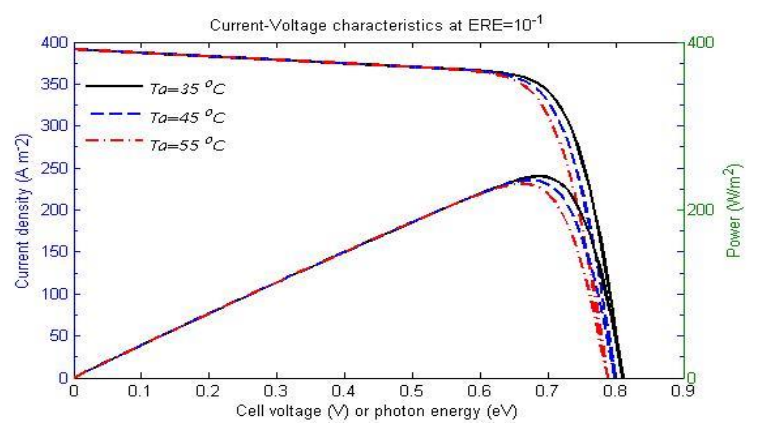

Fig. 6. Voltage-current curve of the solar cell with external radiate efficiency $(E R E)=10-1$ at different temperatures.

Table 5 Energy distribution and corresponding parameters of a c-Si solar cell with external radiate efficiency (ERE) $=10-5$ at different temperatures.

\begin{tabular}{|c|c|c|c|c|c|c|c|c|c|c|c|c|}
\hline \multicolumn{13}{|c|}{ crystalline silicon solar cell, $\mathrm{Eg}_{\mathrm{g}}=1.1246 \mathrm{Ev}$} \\
\hline \multicolumn{13}{|c|}{ ERE $=10^{-5}$} \\
\hline & \multicolumn{4}{|c|}{$\mathrm{Ta}=328.15 \mathrm{~K}$} & \multicolumn{4}{|c|}{$\mathrm{Ta}_{\mathrm{a}}=328.15 \mathrm{~K}$} & \multicolumn{4}{|c|}{$\mathrm{Ta}=328.15 \mathrm{~K}$} \\
\hline & Current & Voltage & Power & $P_{\text {trasidat }}$ & Current & Voltage & Power & $P_{\text {tindedet }}$ & Current & Voltage & Power & $P_{\text {trocideat }}$ \\
\hline Units & $\mathrm{A} / \mathrm{m}^{2}$ & $\mathbf{v}$ & $\mathrm{W} / \mathrm{m}^{2}$ & $\%$ & ${\mathrm{~A} / \mathrm{m}^{2}}^{2}$ & $\mathbf{v}$ & $\mathrm{w} / \mathrm{m}^{2}$ & $\%$ & $\mathrm{~A} / \mathrm{m}^{2}$ & $\mathbf{v}$ & $\mathrm{w} / \mathrm{m}^{2}$ & $\%$ \\
\hline 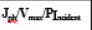 & 436.4 & 1.124 & 1000.25 & 100 & 436.4 & 1.124 & 1000.25 & 100 & 436.4 & 1.124 & 1000.25 & 100 \\
\hline 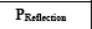 & 43.64 & & 100.0 & 10.00 & 43.64 & & 100.0 & 10.00 & 43.64 & & 100.0 & 10.00 \\
\hline $\mathrm{P}_{\text {Belour }}$ & & & 174.9 & 17.49 & & & 174.9 & 17.49 & & & $\begin{array}{l}174.9 \\
\end{array}$ & 17.49 \\
\hline$P_{\text {Theocontivition }}$ & & & 283.1 & 28.31 & & & 283.1 & 28.31 & & & 283.1 & 28.31 \\
\hline$P_{\text {Eminisina }}$ & 0 & & 0 & 0 & 0 & & 0 & 0 & 0 & & 0 & 0 \\
\hline $\mathbf{P}_{\text {Carot }}$ & & 0.059 & 20.8 & 2.08 & & 0.061 & 21.5 & 2.15 & & 0.063 & 22.2 & 2.22 \\
\hline $\mathbf{P}_{\text {Anglo }}$ & & 0.285 & 99.6 & 9.96 & & 0.294 & 102.9 & 10.29 & & 0.303 & 106.1 & 10.63 \\
\hline$P_{\text {PRRS }}$ & 22.96 & & 25.8 & 2.58 & 23.47 & & 26.3 & 2.63 & 24.34 & & 27.36 & 2.73 \\
\hline$P_{\text {PRR.Y }}$ & & 0.305 & 106.7 & 10.67 & & 0.315 & 110.3 & 11.03 & & 0.325 & 113.8 & 11.38 \\
\hline$P_{\text {Sereire }}$ & & 0.035 & 12.2 & 1.22 & & \begin{tabular}{|l|l|}
0.0348 \\
\end{tabular} & 12.08 & 1.20 & & \begin{tabular}{|l|l|}
0.035 \\
\end{tabular} & 12.25 & 1.22 \\
\hline Pshurt & 20.49 & & 23.03 & 2.30 & 19.62 & & 22.06 & 2.20 & 18.77 & & 21.1 & 2.11 \\
\hline$P_{\text {Output }}$ & $\begin{array}{l}\text { JMPP } \\
=349.3\end{array}$ & $\begin{array}{l}\text { VMPP } \\
=0.457\end{array}$ & 159.62 & $\begin{array}{c}15.95 \\
(\eta)\end{array}$ & \begin{tabular}{|l} 
JMPPP \\
$=-349.66$
\end{tabular} & $\begin{array}{l}\text { VMPPP } \\
=0.436\end{array}$ & 152.48 & $15.24(\eta)$ & \begin{tabular}{|l|l} 
JMPPP \\
$=3449.64$ \\
\end{tabular} & $\begin{array}{l}\begin{array}{l}\text { VMPPP } \\
=0.415\end{array} \\
\end{array}$ & 145.34 & 14.53(n) \\
\hline$\Delta$ & 0.01 & -0.01 & -5.5 & -0.56 & 0.01 & -0.01 & -5.4 & -0.54 & 0.01 & -0.01 & -5.9 & -0.59 \\
\hline$P_{\text {Heat }}$ & & 746.13 & & 74.6 & & 753.14 & & 75.3 & & 760.81 & & 76.08 \\
\hline
\end{tabular}

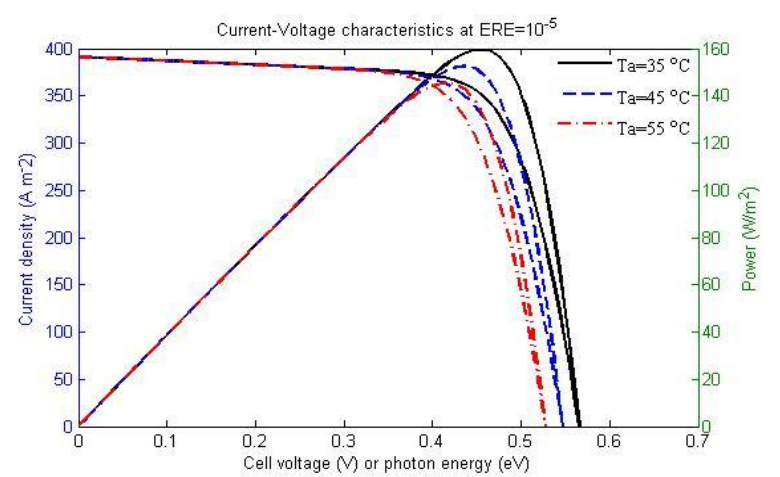

Fig. 7. Voltage-current curve of the solar cell with external radiate efficiency $($ ERE $)=10-1$ at different temperatures.

Table 6 Energy distribution and corresponding parameters of a c-Si solar cell with external radiate efficiency $(\mathrm{ERE})=10-1$ and spectral reflectance $\left(R_{-} c\right)=0.05$ at room temperature.

\begin{tabular}{|c|c|c|c|c|}
\hline \multicolumn{5}{|c|}{ c-Si solar cell, $\mathrm{E}_{\mathrm{g}}=1.1246 \mathrm{eV}$} \\
\hline ERE/Ta/R & $10^{-1}$ & & & 0.05 \\
\hline & Current & Voltage & Power & /PIncident \\
\hline & $\mathrm{A} / \mathrm{m}^{2}$ & $\mathrm{~V}$ & $\mathrm{~W} / \mathrm{m}^{2}$ & $\%$ \\
\hline $\mathrm{J}_{\mathrm{ph}} / \mathrm{V}_{\mathrm{max}} / \mathrm{P}_{\text {Incident }}$ & 436.40 & 1.1246 & 1000.25 & 100 \\
\hline POptical & 21.82 & & 50.01 & 5.00 \\
\hline PBelow & & & 184.62 & 18.48 \\
\hline PThermalization & & & 299.58 & 29.93 \\
\hline PEmission & 0.29 & & 0.33 & 0.03 \\
\hline PCarnot & & 0.057 & 21.45 & 2.14 \\
\hline PAngle & & 0.276 & 102.47 & 10.24 \\
\hline PNRR-J & 11.42 & & 12.84 & 1.28 \\
\hline PNRR-V & & 0.059 & 21.96 & 2.19 \\
\hline PSeries & & 0.037 & 13.78 & 1.37 \\
\hline PShunt & 30.55 & & 34.34 & 3.43 \\
\hline POutput & 371.33 & 0.696 & 258.51 & $25.85(\eta)$ \\
\hline$\Delta$ & -0.01 & 0 & 0.36 & 0.03 \\
\hline PHeat & & 691.04 & & 69.08 \\
\hline
\end{tabular}

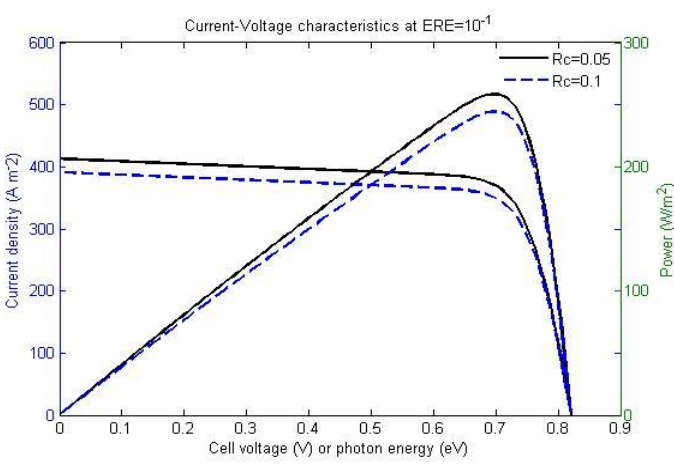

Fig. 8. Voltage-current curve of the solar cell with external radiate efficiency $(E R E)=10-1$ and spectral reflectance $\left(R_{-} c\right)=0.05$ at room temperature.

\section{Conclusion}

This paper study of the intrinsic and external losses in solar cells and the determination of the resulting energy loss at different temperatures. It also discusses the effect of external radiative efficiency and spectral reflectance on the performance of the solar cell. The results show that the processes of loss and temperature increase have a major impact on the efficiency of the solar cell. With the increase of these two factors, the efficiency decreases, and the basic losses processes have the majority of the energy falling and thus the amount of heat more. In addition to the loss processes, the operating temperature and the external radiate efficiency affect the performance of the solar cell.

\section{References}

[1]- Wang, A. and Y. Xuan (2018). "A detailed study on loss processes in solar cells." Energy 144: 490-500.

[2]- Green, M. A., et al. (2019). "Solar cell efficiency tables (version 54)." Progress in Photovoltaics: Research and Applications 27(NREL/JA-5K00-74116).

[3]- Shockley, W. and H. J. Queisser (1961). "Detailed balance limit of efficiency of p-n junction solar cells." Journal of Applied Physics 32(3): 510-519.

[4]- Henry, C. H. (1980). "Limiting efficiencies of ideal single and multiple energy gap terrestrial solar cells." Journal of Applied Physics 51(8): 4494-4500.

[5]- Wurfel .P and W. Ruppel (1980) . "Upper limit of thermophotovoltaic solar-energy conversion," IEEE Trans.Electron Devices 27(4): 745-750.

[6]- Trupke, T., et al. (2003). "Temperature dependence of the radiative recombination coefficient of intrinsic crystalline silicon." Journal of Applied Physics 94(8): 4930-4937.

[7]- Kerr M.J, A. Cuevas, and P. Campbell (2003). "Limiting efficiency of crystalline silicon solar cells due to Coulombenhanced Auger recombination," Progress in Photovoltaics:Research and Applications 11(2):97-104.

[8]- Hirst, L. C. and N. J. Ekins-Daukes (2011). "Fundamental losses in solar cells." Progress in Photovoltaics: Research and Applications 19(3): 286-293.

[9]- Richter, A., et al. (2012). "Improved quantitative description of Auger recombination in crystalline silicon." Physical Review B 86(16): 165202.

[10]- M. Jost and M. Topič (2014). “Efficiency limits in photovoltaics: Case of single junction solar cells," Facta universitatis - series: Electronics and Energetics 27(4):631-638.

[11]- Dupré, O., et al. (2015). "Physics of the temperature coefficients of solar cells." Solar energy materials and solar cells 140: 92-100.

[12]- Dupré,O , R. Vaillon, and M. A. Green(2016). “A full thermal model for photovoltaic devices," Solar Energy140 : 73-82.

[13]- R. Kimovec and M. Topič (2017)."Comparison of measured performance and theoretical limits of gaas laser power converters under monochromatic light," Facta universitatis series: Electronics and Energetics30(1):93-106.

[14]- Ascencio-Vasquez, J., et al. (2019). Application of the full thermal model for PV devices. 